\title{
Transfer of NBS X-ray Beam Calibrations
}

\author{
J. S. Pruitt, A. Allisy, ${ }_{1}^{2}$ G. Joyet, ${ }^{3}$ W. Pohlit, ${ }^{4}$ M. Tubiana, ${ }_{1}^{5}$ and C. Zupančič ${ }^{6}$
}

(January 17, 1962)

\begin{abstract}
A large aluminum ionization chamber has been calibrated at the United States National Bureau of Standards to determine the total amount of energy transported by a bremsstrahlung beam. This chamber was carried to Europe and used to transfer this absolute calibration to similar ionization chambers in betatron laboratories in France, Western Germany, Switzerland, and Yugoslavia. The transfers were made by direct experimental comparison of the chamber sensitivities in the betatron X-ray beams in these laboratories. The transferred calibrations were corrected for differences in X-ray beam size and filtration.
\end{abstract}

\section{Introduction}

The program of the High Energy Radiation Section of the United States National Bureau of Standards (NBS) has for several years included the development of accurate methods of measuring the energy transported by the bremsstrahlung beam from a betatron or an electron synchrotron. The result of this work has been the experimental calibration of a special ionization chamber, of a type labeled P2, so that a measurement of the charge collected during an X-ray exposure serves as an indication of the total beam energy incident on the chamber face.

The value of a calibrated ionization chamber results from its use for transferring these absolute calibrations to other laboratories, by experimentally comparing its sensitivity to that of replica ionization chambers in these laboratories. This procedure eliminates the need for each laboratory to reproduce the original calibration experiments, which would demand a considerable investment of time and money. A laboratory with a calibrated replica chamber has the information required to make its own absolute determination of the total radiation energy incident on experimental apparatus, a number required for quantitative interpretation of experimental results.

The purpose of this report is to describe the transfer of the NBS calibrations to ionization chambers in betatron laboratories in France, Western Germany, Switzerland, and Yugoslavia, on a mission financed jointly by the National Bureau of Standards and the World Health Organization. The transfer work was done with a particular chamber labeled P2-4, which was transported to these laboratories by J. S. Pruitt. By previous arrangement, a replica P2 chamber had been constructed in each country, so that the transfer work in each laboratory was reduced to an experimental comparison of the sensitivities (ionization produced by unit incident energy) of two

\footnotetext{
1 This work was supported in part by the World Health Organization. The paper was written by the senior author, and describes work performed in collaboration with the junior authors.

2 Laboratoire de Dosimétrie, Paris, France.

3 Laboratoire de Dosimétrie, Paris, Fran

${ }^{4}$ Kax Planck Institut für Bionhysik, Frankfurt-am-Main, Western Germany.

${ }^{4}$ Max Planck Institut für Bionhysik, Frankfurt-am-
Institut Gustave-Roussy, Villejuif (Seine) France.

${ }^{6}$ Inštitut Jožef Stefan, Ljubljana, Yugoslavia.
}

similar chambers, plus a determination of any change in the $\mathrm{P} 2-4$ calibration introduced by its use in a different laboratory. The comparisons were made by measuring the relative amount of charge collected from each chamber when they were given identical $\mathrm{X}$-ray exposures, and the calibration changes were obtained from information provided by the NBS, where a study has been made of the effects of changing the beam filtration and beam dimensions.

The P2 chamber and its calibration are described in more detail in the next section. The subsequent sections contain an outline of the comparison techniques and descriptions of the four individual comparisons, listing instrumental details and the results obtained. The last section is a summary of these results.

\section{Background}

The P2 chambers are large, flat, multiplate ionization chambers intended for use with X-ray beams up to $20 \mathrm{~cm}$ in diameter with peak photon energies between 6 and $170 \mathrm{Mev}$, and with intensities between 0.5 and $1,000 \mu \mathrm{w} / \mathrm{cm}^{2}$. Figure 1 is a schematic cross section of one of these chambers, figure 2 is an exterior view, and figure 3 shows the ionization collection region, after the outer shell and the thick front wall have been removed. The internal plates and the front and back walls of this chamber are made of 2024 Dural, an aluminum alloy whose composition is listed in table 1 . The thickness of the front wall $(9.4 \mathrm{~cm})$ was chosen to minimize the variation of chamber calibration with X-ray beam peak energy for a filtration of $4.5 \mathrm{~g} / \mathrm{cm}^{2}$ of aluminum. The total thickness of the air gap is $5 \mathrm{~cm}$, large enough to provide high sensitivity. It is divided into 12 sectors to reduce the probability of ion recombination and to prevent excessive loss of ionization from electrons escaping from the periphery.

The calibration was performed with two instruments which determine the total energy transported by photons in an exposure to an $\mathrm{X}$-ray beam of small cross section. These are a refined version of the Laughlin lead calorimeter [1], ${ }^{7}$ and a $\mathrm{NaI}(\mathrm{Tl})$ crystal spectrometer [2]. The experimental calibra-

\footnotetext{
7 Figures in brackets indicate the literature references at the end of this paper
} 
HN CONNECTOR

HIGH VOLTAGE

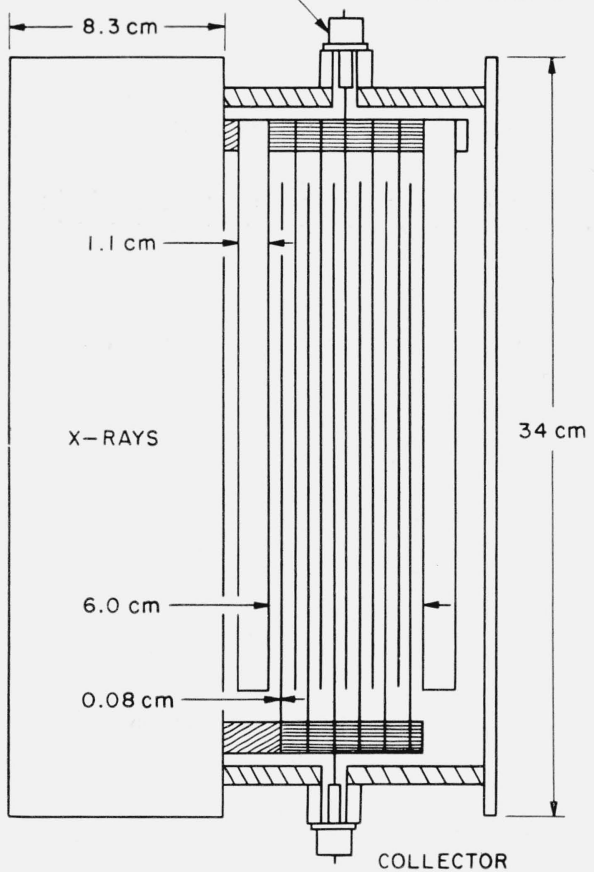

\section{\#IL KEL-F \\ 韭 STEEL \\ III BRASS}

REMAINING PARTS-2024 DURAL

Figure 1. Schematic cross section of $P \mathcal{2}$ ionization chamber.

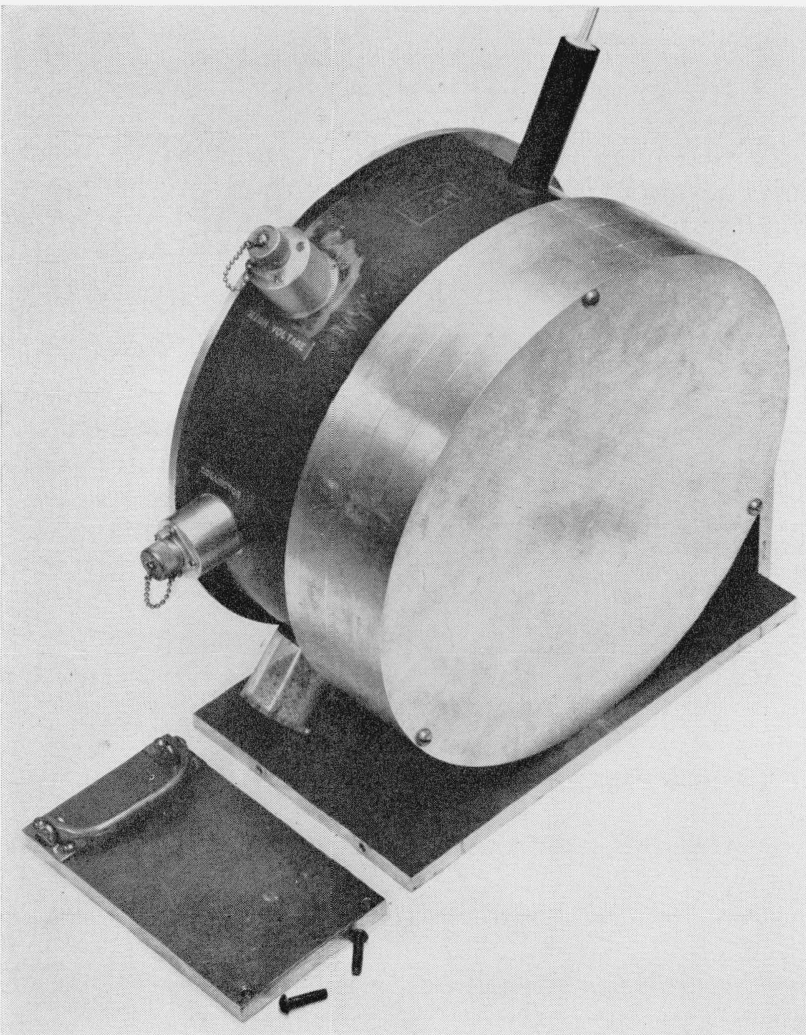

Figure 2. Exterior view of P\& ionization chamber. tion of the chamber taken to Europe, P2-4, is listed in units of joules/coulomb as $\mathrm{Cal}(\mathrm{P} 2-4)$ in table 2, and is plotted in figure 4 . These numbers were obtained in a series of four experiments [3]. Those from experiments a and $c$ have estimated errors of \pm 3 percent and \pm 2 percent respectively. Those from experiments $b$ and $d$ were combined to yield a calorimetric calibration with a conservatively estimated error of \pm 2 percent. They refer to measurements in an X-ray beam $4.2 \mathrm{~cm}$ in diameter, filtered by $4.5 \mathrm{~g} / \mathrm{cm}^{2}$ of a low atomic number material like aluminum, in dry air at a temperature of $22{ }^{\circ} \mathrm{C}$ and a pressure of $760 \mathrm{~mm}$ of mercury.

The calibration of $\mathrm{P} 2-4$ varies to some extent with the filtration and diameter of the incident X-ray beam. These variations have been studied in detail at the NBS and are described in another report [4]. They are less than 2 percent for all of the changes in experimental conditions encountered in the intercomparisons described herein.

TABLE 1. Elemental composition of 2024 Dural

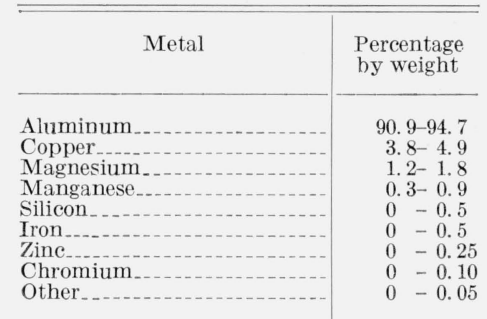

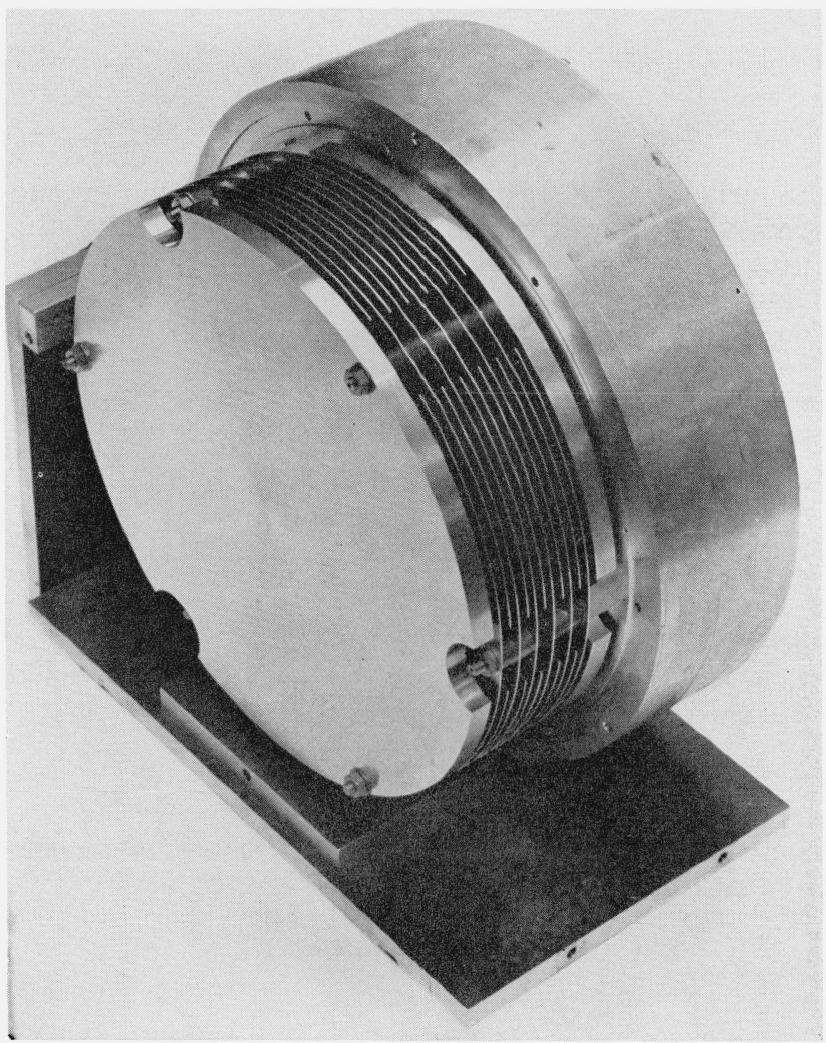

Figure 3. Internal structure of $P^{2}$ ionization chamber. 
Table 2. Calibration of $P 2-4$ in an $X$-ray beam of $4.2 \mathrm{~cm}$ in diameter, filtered by $4.5 \mathrm{~g} / \mathrm{cm}^{2}$ of aluminum, at $20{ }^{\circ} \mathrm{C}$ and $760 \mathrm{~mm}$ of mercury

\begin{tabular}{|c|c|c|c|}
\hline \multicolumn{2}{|c|}{ Spectrometer experiments } & \multicolumn{2}{|c|}{ Calorimeter experiments } \\
\hline Peak energy & $\mathrm{Cal}(\mathrm{P} 2-4)$ & Peak energy & $\mathrm{Cal}(\mathrm{P} 2-4)$ \\
\hline $\begin{array}{l}\text { Mev } \\
\text { (a) } 19.6 \\
24.6 \\
29.6 \\
34.6 \\
39.6 \\
44.7 \\
49.7 \\
59.7\end{array}$ & $\begin{array}{l}\text { Joules/coulomb } \\
4.04 \times 10^{5} \\
4.14 \\
4.10 \\
4.10 \\
4.07 \\
3.96 \\
3.86 \\
3.82\end{array}$ & $\begin{array}{c}\text { Mev } \\
\text { (b) } 18.2 \\
19.8 \\
21.7 \\
25.9 \\
31.3 \\
36.7 \\
42.1\end{array}$ & 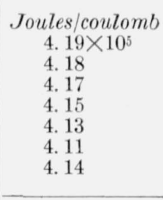 \\
\hline $\begin{array}{r}69.8 \\
89.8 \\
109.9 \\
130.0 \\
150.1 \\
170.2\end{array}$ & $\begin{array}{l}\text { 3. } 73 \\
3.71 \\
3.60 \\
3.64 \\
3.69 \\
3.71\end{array}$ & (d) $\begin{array}{r}20 \\
25 \\
30 \\
35 \\
40 \\
45\end{array}$ & $\begin{array}{l}\text { 4. } 12 \times 10^{5} \\
\text { 4. } 08 \\
\text { 4. } 11 \\
\text { 4. } 10 \\
\text { 4. } 05 \\
\text { 4. } 02\end{array}$ \\
\hline $\begin{array}{r}\text { (c) } 6 \\
8 \\
10 \\
13 \\
16 \\
19\end{array}$ & $\begin{array}{l}\text { 4. } 10 \times 10^{5} \\
4.17 \\
3.99 \\
4.07 \\
4.09 \\
4.17\end{array}$ & $\begin{array}{r}50 \\
60 \\
70 \\
90 \\
110 \\
130 \\
150 \\
170\end{array}$ & $\begin{array}{l}3.99 \\
3.94 \\
3.86 \\
3.80 \\
3.84 \\
3.81 \\
3.82 \\
3.87\end{array}$ \\
\hline
\end{tabular}

a J. E. Leiss, R. A. Schrack and J. S. Pruitt (1957).

b. J. S. Pruitt and S. R. Domen (1958)

c E. G. Fuller and E. Hayward (1959).

d J. S. Pruitt and S. R. Domen (1959).



Figure 4. Calibration of $\mathrm{P}^{2}-4$ at $20^{\circ} \mathrm{C}$ and $760 \mathrm{~mm}$ of mercury in a $4.2-\mathrm{cm}$ diameter bremsstrahlung beam filter by $4.5 \mathrm{~g} / \mathrm{cm}^{2}$ of aluminum.

\section{Comparison Techniques}

Figure 5 shows the general arrangement of physical and electrical components used in the chamber comparisons. The P2 chambers were bombarded by a betatron X-ray beam of small cross section, and the ionization produced during the exposure was measured with the electrometer and associated equipment shown at the right. A monitor was used to obtain a relative measure of the total beam energy during each exposure, and the comparison consisted of the measurement of the ionization per unit monitor reading for each P2 chamber. Systematic errors were minimized by placing the $\mathrm{P} 2$ chambers in identical positions in the X-ray beam, and using the same measuring equipment with each. The X-ray exposures were also the same for the two chambers, where this quantity could be controlled.

The P2 chambers were used with a source of high voltage, $E_{I}$, an electrometer, a polystyrene

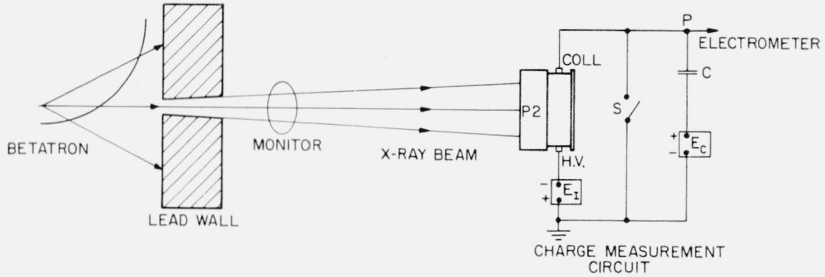

FiguRE 5. Schematic experimental arrangement for chamber comparisons.

capacitor, $C$, and, where possible, a variable source of compensating voltage, $E_{C}$. At the start of each exposure, point $P$ was grounded with the switch $S$ (fig. 5) closed and $E_{C}=0$. After $S$ had been opened and the chamber had been exposed to X-rays, point $P$ was returned to ground potential by varying $E_{C}$ until the electrometer reading returned to its initial value. The ionization charge collected during the X-ray exposure was then simply given by:

$$
q(\text { coulombs })=C(\text { farads }) \times E_{C}(\text { volts }) .
$$

Where it was not feasible to use a source of compensating voltage (Switzerland and Yugoslavia), the electrometer was used as a deflection instrument to indicate the final voltage of point $P, V_{P}$. In these cases the collected ionization charge was given by:

$$
q=C^{\prime} V_{P}
$$

where $C^{\prime}$ is the sum of $C$ and the capacitance of the P2 chamber (about $0.0016 \mu f)$, plus small terms involving the input capacitance of the electrometer, the leakage capacitance to ground, and the electrometer gain. This method of measuring $q$ is less attractive when absolute measurements are required, but it is just as good for relative measurements unless the difference between the capacitances of the P2 chambers being compared is a significant fraction of $C^{\prime}$. Since only relative measurements were required for the chamber comparisons, there was no attempt to make absolute measurements of any of these capacitances.

All of the instrumentation for each comparison, the monitor, voltage sources, electrometer, and capacitor $C$, was supplied by the laboratory where the measurements were made. The instrumental details differed in the different countries, and are described in the following sections.

\section{France}

The French P2 ionization chamber (P2-5) was constructed from the NBS drawings under the direction of A. Allisy. It was compared with the NBS P2-4 in the X-ray beam of the $22.5 \mathrm{Mev}$ Allis-Chalmers betatron at the Institut Gustave Roussy, under the direction of M. Tubiana.

The comparison was made 1 meter from the X-ray source, a standard position for patient irradiation and experimental dosimetry in this laboratory. The beam was circular in cross section, with a diameter of $3.8 \mathrm{~cm}$ at the chamber face. It was 
filtered by the donut wall, the monitor, and an aluminum compensating cone used to flatten the X-ray field, a total average filtration equivalent to about $39 \mathrm{~g} / \mathrm{cm}^{2}$ of aluminum.

The monitor was a flat ionization chamber which is permanently mounted in the X-ray beam. The monitor ionization was measured with a highly stabilized electronic integrating circuit, which automatically shut off the X-ray beam after a fixed and predetermined amount of charge had been collected. This feature assured that the X-ray exposures were identical during each set of runs.

The electrometer was supplied by A. Allisy, and is identical with one he has used to compare X-ray standards at lower energies [5], except for the recent addition of an electronically stabilized power supply. This electrometer was used as a null instrument, with a $0.1 \mu \mathrm{f}$ polystyrene capacitor, and with an electronic power supply for the compensating vcltage. The compensating voltage was continuously adjusted during each exposure to maintain the electrometer input near ground potential. Its final value (of the order of $8.5 \mathrm{v}$ ) was precisely measured with a potentiometer and a voltage divider after X-rays had been shut off, and was taken as a relative measure of the $\mathrm{P} 2$ ionization produced during that exposure.

$E_{I}$ for the P2 chamber was $-1,000 \mathrm{v}$, and was obtained from an electronic power supply.

$\mathrm{P} 2-4$ and $\mathrm{P} 2-5$ were compared in X-ray beams with peak energies of 18 and $22 \mathrm{Mev}$. The results obtained from several comparisons are listed below. Each number represents the average of at least five X-ray exposures:

\begin{tabular}{|c|c|c|c|c|c|}
\hline Mev & \multicolumn{3}{|c|}{22} & \multicolumn{2}{|c|}{18} \\
\hline Sensitivity (P2-5) & \multirow{2}{*}{ 1. 004} & \multirow{2}{*}{ 1. 007} & \multirow{2}{*}{ 1. 006} & \multirow{2}{*}{ 1. 004} & \multirow{2}{*}{ 1. 008} \\
\hline Sensitivity (P2-4) & & & & & \\
\hline
\end{tabular}

\section{Western Germany}

The West German P2 chamber (P2-6) was constructed from the NBS drawings under the direction of W. Pohlit, of the Max Planck Institut für Biophysik. It was compared with $\mathrm{P} 2-4$ in the $\mathrm{X}$-ray beam from the $35 \mathrm{Mev}$ Siemens betatron at that laboratory.

The comparisons were performed with the P2 chambers 1 meter from the source of X-rays, and were made with an X-ray beam which was $4 \mathrm{~cm}$ in diameter at the chamber face. The beam was filtered by a thin glass window in the donut wall, and by the monitor, for a total filtration of about 1 $\mathrm{g} / \mathrm{cm}^{2}$ of aluminum. During the last day of experimentation, several millimeters of lead were added in the beam. Within the limits of error of these comparisons $( \pm 0.2 \%)$, the presence of this extra filter did not affect the ratio of the sensitivities of P2-4 and P2-6, although it is known from experi- ments at the NBS to affect the absolute calibration of $\mathrm{P} 2-4$.

The electrometer was a 2-terminal vibrating reed instrument of German manufacture. It was used as a null instrument, with a $0.02 \mu \mathrm{f}$ polystyrene capacitor and a battery supply of compensating voltage. The compensating voltage at the end of each exposure was of the order of 100 and was measured with a precision voltmeter.

The monitor was a flat ionization chamber with three $1-\mathrm{mm}$ thick metal coated plastic walls. The monitor ionization was measured with a second vibrating reed electrometer, using a circuit similar to the P2 ionization measuring circuit.

$E_{I}$ for the P2 chambers was $-1,200 \mathrm{v}$, obtained from a battery power source.

P2-4 and P2-6 were compared with X-ray beams with peak energies of 20 and $34.5 \mathrm{Mev}$, and the results are listed below. Each number is the average of at least 20 exposures:

\begin{tabular}{|c|c|c|}
\hline Mev & 34.5 & 20 \\
\hline$\frac{\text { Sensitivity (P2-6) }}{\text { Sensitivity (P2-4) }}$ & $\begin{array}{lll}1.000 & 1.003 & 0.999\end{array}$ & $\begin{array}{llll}0.996 & 1.000 & 1.000\end{array}$ \\
\hline
\end{tabular}

\section{Switzerland}

The Swiss P2 chamber (P2-7) was constructed from the NBS drawings under the direction of $\mathrm{G}$. Joyet, of the Betatron and Isotopes Laboratory of the Radiological Department of the Zürich Kantonsspital. It was compared with the NBS P2-4 chamber in the X-ray beam from the $35 \mathrm{Mev}$ Brown Boveri betatron in this hospital.

During the comparison, the chambers were positioned $1 \mathrm{~m}$ from the X-ray source, a position commonly used in this laboratory for patient irradiation and experimental dosimetry. The X-ray beam was rectangular in cross section, and measured $8 \times 12 \mathrm{~cm}$ at the face of the chamber. It was filtered by the donut wall and a copper compensating cone used to flatten the X-ray field. The donut wall is equivalent to about $3.0 \mathrm{~g} / \mathrm{cm}^{2}$ of aluminum and the average thickness of the compensating cone is about $16 \mathrm{~g} / \mathrm{cm}^{2}$ of copper.

The monitor was a $25 \mathrm{r}$ Victoreen r-thimble, mounted to one side of the X-ray beam in a hole in the lead wall.

The electrometer was a 2-terminal vibrating reed model of English manufacture. It was used to indicate the voltage across the $1.6 \mu \mathrm{f}$ polystyrene capacitor charged by the chamber, rather than as a null instrument.

The P2 chamber voltage was $-1,290 \mathrm{v}$, which was obtained from a battery power supply.

P2-4 and P2-7 were compared with a $31 \mathrm{Mev}$ $\mathrm{X}$-ray beam. The exposures were quite long, because of the relative insensitivity of the r-thimble in its position out of the direct beam. It was necessary 
to make 15 partial exposures, each approximately $1 \mathrm{v}$ on the electrometer, before the r-thimble reading was large enough to insure reproducibility (about 17.5 on the r-meter scale). The results of two sets of data taken on successive days are shown below. The first number represents three exposures and the second nine exposures:

\begin{tabular}{c|cc}
\hline \hline Mev & \multicolumn{2}{|c}{31} \\
\hline$\frac{\text { Sensitivity (P2-7) }}{\text { Sensitivity (P2-4) }}$ & 1.007 & 1.011 \\
\hline
\end{tabular}

\section{Yugoslavia}

The Yugoslav P2 chamber (P2-8) was constructed from the NBS drawings under the direction of $\mathrm{Mr}$. Jan, of the Inštitut Jožef Stefan. It was compared with the NBS P2-4 chamber in the $\mathrm{X}$-ray beam from the $35 \mathrm{Mev}$ Brown Boveri betatron at that institute, under the direction of C. Zupančič.

The chambers were compared at a point $180 \mathrm{~cm}$ from the X-ray source, in a beam of circular cross section, $3.9 \mathrm{~cm}$ in diameter. This beam was filtered by the donut wall and the monitor, a total filtration equivalent to about $3.5 \mathrm{~g} / \mathrm{cm}^{2}$ of aluminum.

The electrometer was a current integrator built in that laboratory. It was used with a $4 \mu \mathrm{f}$ capacitor, and was arranged to discharge the capacitor and actuate a mechanical counter when the capacitor charge reached a fixed and predetermined value. The number of counts was taken as a relative measure of the P2 ionization during each exposure.

The monitor was a cylindrical ionization chamber, $10 \mathrm{~cm}$ in diameter and $11.8 \mathrm{~cm}$ long, with $0.1 \mathrm{~cm}$ thick aluminum walls. The monitor ionization was measured with a second, similar, current integrator.

High voltage for the P2 chambers was obtained from a highly stabilized electronic power supply, and was of the order of $-1,200$ volts.

P2-4 and P2-8 were first compared in an X-ray beam with peak photon energy of $27.3 \mathrm{Mev}$, and then in a $21.3 \mathrm{Mev}$ beam. The $\mathrm{P} 2-8 / \mathrm{P} 2-4$ ratios at these two energies were so disparate (they differed by $2 \%$ ), that the ratio was measured at several other energies. The comparisons were made over a period of several days, and some repeat measurements were made at 27.3 and $21.3 \mathrm{Mev}$ during that time, to check the reproducibility. The experimental results are listed below, where each number represents the average of at least 10 exposures:
The sensitivity ratio varies erratically with energy, and the variation is considerably larger than would be expected on the basis of the reproducibility tests. It is difficult to believe that this irregular variation is real, and it is tempting to ascribe it to systematic instrumental errors, which are imperfectly understood. Unfortunately, it was necessary to return the P2-4 chamber to the United States before this question could be resolved, so the ratio of chamber calibrations in the $3.9 \mathrm{~cm}$ Ljubljana betatron beam was taken to be the average value over this energy range:

$\frac{\text { Sensitivity }(\mathrm{P} 2-8)}{\text { Sensitivity }(\mathrm{P} 2-4)}=0.973$ between 18.3 and $30.3 \mathrm{Mev}$.

\section{Comments}

The experimental conditions for these comparisons and the comparison results are summarized in table 3. Each number in the fifth column is the ratio of the European chamber sensitivity in the local $\mathrm{X}$-ray beam (in coulomb/joule) to the P2-4 sensitivity in the same beam.

The European chambers differ from P2-4 for several reasons. Part of the difference can be attributed to small differences in dimensions, which are difficult to control to the accuracy required to produce an exact replica. For instance, the measured thickness of the central region of the front wall of P2-5 is $0.04 \mathrm{~cm}$ less than that of $\mathrm{P} 2-4$, and the total thickness of the air gap is $0.01 \mathrm{~cm}$ larger. These differences are large enough to explain the 0.6 percent increase in sensitivity of $\mathrm{P} 2-5$ near $20 \mathrm{Mev}$. The reduced sensitivity of $\mathrm{P} 2-8$, on the other hand, is caused by the use of internal plates which are 1 $\mathrm{mm}$ thick, instead of $0.8 \mathrm{~mm}$, as specified in the chamber plans. This change should reduce its air gap thickness, and consequently, its calibration, by about 2.5 percent, in approximate agreement with the average measured reduction. The enhanced sensitivity of $\mathrm{P} 2-7$ is probably caused by a different kind of change, the use of an aluminum alloy which is considerably purer than 2024 Dural. The alloy used contains no copper, and very little metal of high atomic number $(\langle 1 \%$ of $\mathrm{Mn})$. Although it is difficult to analyze quantitatively the effects of a change in the atomic number of the alloy, tests performed at the NBS indicate that a decrease in atomic number will increase the sensitivity in this energy region [4].

The absolute calibration of each of these replica chambers in the X-ray beam used for the intercomparison can be obtained by dividing the P2-4 calibration of figure 3 (in joules/coulomb) by the

\begin{tabular}{|c|c|c|c|c|c|c|c|c|c|c|}
\hline Mev & 18. 3 & & 21. 3 & & 24.3 & \multicolumn{4}{|c|}{27.3} & 30.3 \\
\hline$\frac{\text { Sensitivity (P2-8) }}{\text { Sensitivity (P2-4) }}$ & 0.971 & 0. 980 & 0.978 & 0.974 & 0.987 & 0.961 & 0. 956 & 0.955 & 0. 966 & 0. 971 \\
\hline
\end{tabular}


TABLE 3. Calibrations of European chambers

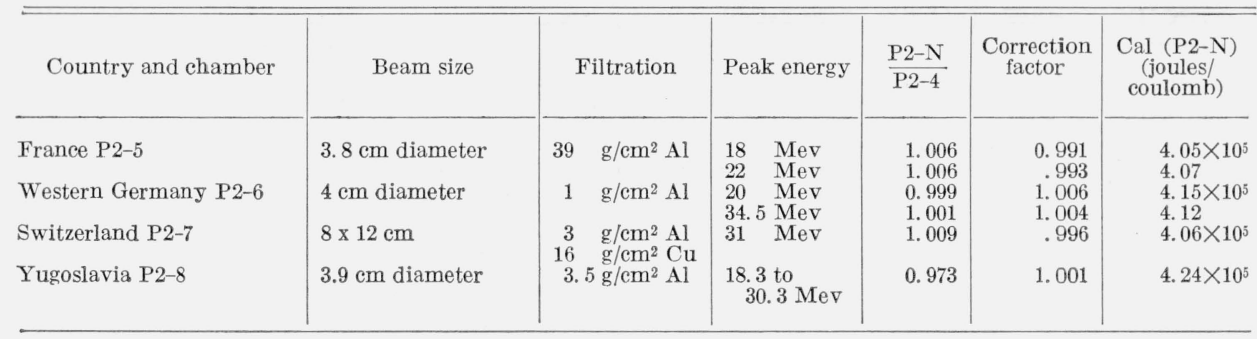

sensitivity ratio of table 3 and correcting for differences in beam diameter and filtration. These corrections were obtained from the aforementioned report [4], and are listed as multiplicative factors in table 3 along with the final calibration of each replica chamber in the local X-ray beam.

The authors express their appreciation to the many people who helped us make these comparison measurements, and to H. O. Wyckoff of the NBS for suggesting the comparisons and for helping with the arrangements.

(Paper 66C2-91)

\section{References}

[1] J. S. Laughlin and J. W. Beattie, Calorimetric determination of the energy flux of 22.5 Mev X-rays, Rev. Sci. Inst. 22, 572 (1951).

[2] H. W. Koch, J. E. Leiss, and J. S. Pruitt, Crystal spectrometer calibration of a high energy X-ray intensity monitor, Bull. APS II 1, 199 (1956) A

[3] Descriptions of these experiments will be published soon. The earliest calorimeter experiment was described by: J. S. Pruitt and S. R. Domen, Calorimetric X-ray monitor calibration at betatron energies, Rad. Research [A] 9, 168 (1958).

[4] J. S. Pruitt and S. R. Domen, Determination of total $\mathrm{X}$-ray beam energy with a calibrated ionization chamber (to be published as an NBS Monograph).

[5] A. Allisy, L. DeLaVergne, and H. O. Wyckoff, Intercomparison of French and U.S. Roentgen Ray Standards, Acta Rad. 48, 484 (1957). 